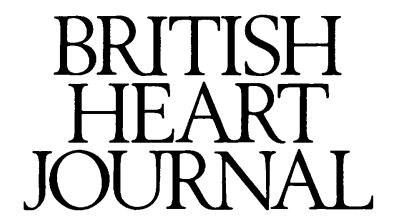

As early as 1923 it was recognised that quinidine could cause sudden death, ${ }^{1}$ but it was not until 1964 that the cause of quinidine syncope was unequivocally established as a form of paroxysmal ventricular arrhythmia. ${ }^{2}$ In 1966 Dessertenne of Paris reported his fundamental observations on the characteristics of the arrhythmia and proposed the term torsade de pointes. ${ }^{3}$ (Even now there is inconsistency in French publications about the plural usage.) He noted an association with bradycardia and that quinidine and similar antiarrhythmic drugs could induce the arrhythmia and were associated with a high mortality when used for its management. The arrhythmia seemed to be an idiosyncratic reaction to therapeutic or sub-therapeutic doses of the drug and not a manifestation of toxicity.

Although it is 16 years since the British Heart Journal first drew the attention of English speaking cardiologists to torsade de pointes, ${ }^{4}$ it had been briefly discussed two years earlier ${ }^{5}$; indeed, while isolated instances of this arrhythmia had been reported previously in English language journals, its nature and the circumstances surrounding its occurrence had been poorly described. This is no longer the case and there are now extensive publications from many countries. Effective treatment is available for most varieties of this condition, but it is not clear that the clinical recognition of the arrhythmia, and therefore its management, has generally improved. Although understanding of the arrhythmia at a practical, clinical level has increased, the basic electrophysiological mechanisms underlying torsade de pointes remain obscure despite a decade and a half of research.

\section{Definition and diagnosis}

The essential features of the diagnosis are of a variant of paroxysmal ventricular tachycardia associated with prolongation of the QT interval (more accurately the JT interval) or prominent $U$ waves ${ }^{3}$ which may be hard to distinguish from the end of a long $\mathrm{T}$ wave. ${ }^{6} \mathrm{However}, \mathrm{QT}$ prolongation or $U$ waves may appear only briefly before the onset of the arrhythmia. Typically the QRS axis changes direction in an undulating fashion during paroxysms. Occasionally paroxysms appear uniform. Episodes may be brief, consisting of only a few complexes, or prolonged when they may degenerate into ventricular fibrillation. Between episodes pauses are also often observed on the electrocardiogram. ${ }^{3}$

Underlying mechanisms of arrhythmia

Clinically, torsade de pointes is a heterogeneous condition and there is no reason why the basic mechanisms under- lying the arrhythmia might not also vary. However, there are several unifying features on the electrocardiogram that suggest that a common substrate may exist.

The response to sympathetic stimuli identifies two main types of torsade de pointes. One is associated with bradycardia and ventricular pauses and improves with sympathetic stimulation, which increases the ventricular rate. This form tends to occur in older patients who are more likely to have conduction defects and perhaps also to receive antiarrhythmic agents. The other is precipitated by increased sympathetic activity. This tends to occur in younger subjects and is sometimes associated with sensorineural deafness. ${ }^{78}$ Episodes of bradycardia may also be seen between events in these patients.

\section{Possible role of reentry}

The propagation of the activation wavefront, the action potential duration, and the process of repolarisation are asynchronous events. There are several gradients within the heart, with the time for repolarisation being shorter near the apex than near the base and shorter near the epicardium than near the endocardium, which accounts for the fact that QRS and T wave polarity are usually the same. ${ }^{9}$ The QT interval encompasses the time from first activation of the ventricular myocardium to the final repolarisation. Thus the QT interval may be prolonged if any of the above three phases is delayed. Regional changes in the above events will lead to regional variations (dispersion) in the refractory period, predisposing to reentry arrhythmias. Such arrhythmias may propagate in irregular patterns as areas with prolonged refractory periods are encountered which act as shifting inexcitable island barriers to the progress of ventricular activation. Both bradycardia and quinidine-like agents can exacerbate the heterogeneity of repolarisation and increase the likelihood of reentrant arrhythmias. ${ }^{10}$ However, if torsade de pointes were due to reentry a sustained ventricular tachycardia of this type should be inducible by an electrical stimulus during the TU wave, which was not the case at least in our early experience, ${ }^{11}$ and subsequently that of others. This is strong evidence against the reentry theory.

\section{Possible role of "chaotic" behaviour}

More recently, chaos theory has been applied to the study of ventricular arrhythmias. In common parlance chaos refers to disorder. However, scientists have appropriated the term to indicate the underlying orderliness of apparent disorder. ${ }^{12}$ Thus true random "noise" in any system is not 
"chaos". In the dynamics of chaos, two initial conditions that are very similar will eventually behave very differently as time proceeds owing to subtle interactions with their environments. There are definite rules underlying this process, which can be detected and defined by mathematical equations.

The reason for alterations in a stable heart rhythm might be changes in electrolyte concentrations, variations in autonomic tone, or myocardial ischaemia. Any of these perturbations will alter the behaviour of the myocardium and the conduction system and will also have effects on other systems that modulate cardiac activity. Ultimately the perturbation will feed back on itself (reinforcing or diminishing the effects of the original intervention), closing the loop. For instance, myocardial ischaemia may lead to increased sympathetic activity and hence an increase in heart rate and contractility which in turn may aggravate the ischaemia. At the moment chaos theory is largely concerned with detecting the presence of such interactions but has not advanced far enough to predict the outcome.

In torsade de pointes, ventricular pauses or imbalances in autonomic tone could act as perturbations in a normally stable rhythm. Although models of torsade de pointes have not been studied specifically for evidence of chaos, ventricular tachycardia and fibrillation have. ${ }^{13}$ In these models there is evidence of chaotic behaviour before the appearance of ventricular fibrillation, but ventricular fibrillation was not itself thought to be chaotic. The perturbations which might lead to chaos in these models also lead to an ideal substrate for reentry arrhythmias, and these, rather than a true chaotic system, seem responsible for ventricular tachycardia and its degeneration to fibrillation.

Chaos in the technical sense is not necessarily unstable, and indeed it is likely that chaos is a healthy state in most biological systems. ${ }^{14}$ For instance the heart rate varies continually under the influence of the autonomic nervous system which itself changes the input to the heart according to various factors including the level of mental, emotional, and physical activity; respiratory phase; and blood pressure. When cardiac disease supervenes (for example, heart failure) the heart rate becomes fixed, the normal chaotic behaviour is lost, and the risk of sudden cardiac death increases. $^{15}$

\section{Possible role of early afterdepolarisations}

As defined by Cranefield and Aronson, ${ }^{16}$ an early afterdepolarisation is a depolarising afterpotential that begins before repolarisation would normally be complete. Lately, the idea that the $U$ waves represent early afterdepolarisations has gained ground. ${ }^{17}$ At the cellular level the rate of repolarisation depends on the rapidity with which potassium is ejected from the cell, which far outweighs the usual slower continued influx of calcium and sodium. ${ }^{18}$ However, if the egress of potassium is slowed or halted, ${ }^{18} 19$ the inward flow of calcium and sodium will slow or reverse the normal repolarisation of the cell thus producing early afterdepolarisations. If the early afterdepolarisations are sufficiently large they may trigger further action potentials and themselves lead to the production of arrhythmias. Caesium by blocking potassium repolarising currents can provoke early afterdepolarisations, QT(U) abnormalities, and arrhythmias and thus produce a condition identical with torsade de pointes. ${ }^{20}$

Several observations on the clinical setting of torsade de pointes support a role for early afterdepolarisations. Electrolyte disturbances can cause prolongation of the QT interval. Hypokalaemia and hypomagnesaemia both increase the QT(U) interval and early afterdepolarisations and may provoke torsade de pointes, while magnesium given intravenously may suppress torsade de pointes even in the absence of hypomagnesaemia. ${ }^{21}$ Indeed, a case of what was clearly torsade de pointes responding promptly to parenteral magnesium was reported over 50 years ago. ${ }^{22}$ However, acute potassium supplementation does not seem to be effective even when the serum potassium is low. ${ }^{23}$ Hypocalcaemia causes QT prolongation, mainly by increasing ST segment prolongation rather than TU wave prolongation, and it has not been implicated as a cause of torsade de pointes. ${ }^{24}$ Bradycardia provokes early afterdepolarisations, especially in the presence of low extracellular potassium concentrations and in the presence of low concentrations of quinidine.$^{18}$ Bradycardia may facilitate a hypokalaemic micro-environment around the cell because a prolonged diastole would allow the sodium/potassium pump to operate for an extended period. Caesium blocks potassium channels during the repolarisation phase of the action potential and can accentuate early afterdepolarisations and provoke arrhythmias. ${ }^{25}$ This is especially likely if the sodium channel has not been blocked by tetrodoxin.

Sympathetic stimuli can increase not only late but also early afterdepolarisations in animal models of torsade de pointes, ${ }^{2627}$ probably by enhancing inward sodium and calcium currents during repolarisation or by inducing local hypokalaemia by stimulating cellular potassium uptake. The occurrence of familial forms of the disease, often associated with sensorineural deafness, fits better with a general disturbance of cardiac myocyte function rather than a reentry mechanism..$^{28}$ Patients susceptible to torsade de pointes due to one class of quinidine-like antiarrhythmic agents may develop torsade de pointes when given another such agent. Occurrence of torsade de pointes with the related antimicrobial agents erythromycin and pentamidine $^{29} 30$ (the latter now used for complications of AIDS) further supports a generalised disturbance of cell function.

Our understanding of torsade de pointes has expanded considerably over the past 16 years. Basic science has developed models of the condition that reveal the underlying electrophysiological disorder which might help predict the likely effect of new drugs.

Although the direct experimental evidence is not conclusive the circumstantial clinical evidence points strongly towards a role for early afterdepolarisations in the genesis of torsade de pointes, which may not be so far from Dessertenne's original theory of alternating ventricular foci. Indeed, Dessertenne may have coined what is now a relevant phrase with his reference to "order and chaos in electrocardiography". ${ }^{31}$

JOHN G F CLELAND

Cardiovascular Division,

Royal Postgraduate Medical School,

Hammersmith Hospital,

Du Cane Road,

London W12 ONN

1 Viko LE, Marvin HM, White PD. Clinical report of the use of quinidine sulfate. Arch Intern Med 1923;31:345-63.

2 Selzer A, Wray WH. Quinidine syncope: paroxysmal ventricular fibrillation occurring during treatment of chronic atrial arrhythmias. Circulation 1964;30:17-26.

3 Dessertenne F. La tachycardie ventriculaire à deux foyers opposés variables. Arch Mal Coeur 1966;59:263-72.

4 Krikler DM, Curry PVL. Torsade de pointes, an atypical ventricular tachycardia. Br Heart J 1976;38:117-20.

5 Krikler DM. A fresh look at cardiac arrhythmias. Pathogenesis and presentation. Lancet 1974;i:913-8.

6 Ejvinsson G, Orinius E. Prodromal ventricular premature beats preceded by a diastolic wave. Acta Med Scand 1980;208:445-50.

7 Jervell A, Lange-Nielsen F. Congenital deaf-mutism, functional heart disease with prolongation of the QT-interval, and sudden death. Am Heart J 1957;54:59-68.

8 Schwartz PJ, Periti M, Malliani A. The long QT syndrome. Am Heart J 1975;89:378-90.

( Burgess MJ Green LS, Millar K, et al. The sequence of normal ventricular recovery. Am Heart J 1972;84:660-9. 
10 Han J, Millet D, Chizzonitti B, Moe GK. Temporal dispersion of recovery of excitability in atrium and ventricle as a function of heart rate. Am Heart $\mathrm{J}$ 1966;71:481-7.

11 Evans TR, Curry PVL, Fitchett DH, Krikler DM. "Torsade de pointes" initiated by electrical ventricular stimulation. J Electrocardiol 1976;9: 255-8.

12 Gleick J. Chaos: making a new science. New York: Viking Penguin, 1987

13 Kaplan DT, Cohen RJ Is fibrillation chaos? Circ Res 1990;67:886-92.

14 Winfree AT. Sudden cardiac death: a problem in topology. Sci Am 1983 248:118-31.

15 Goldberger AL. Non-linear dynamics in heart failure: implications of long wavelength cardiopulmonary oscillations. Am Heart J 1984;107:612-5.

16 Cranefield PF, Aronson RS. Cardiac arrhythmias: the role of triggered activity and other mechanisms. New York: Futura Publishing, 1988:12.

17 El-Sherif N, Bekheit SS, Henkin R. Quinidine-induced long QTU interva and torsade de pointes: role of bradycardia-dependent early afterdepolarisations. J Am Coll Cardiol 1989;14:252-7.

18 Roden DM, Hoffman BF. Action potential prolongation and induction of abnormal automaticity by low quinidine concentrations in canine Purkinje fibers. Relationship to potassium and cycle length. Circ Res 1985;56: 857-67.

19 Corabeouf E, Deroubaix E, Coulombe A. Acidosis induced abnormal repolarizations and repetitive activity in isolated dog Purkinje fibres. J Physiol (Paris) 1980;76:97-106.

20 Levine JW, Spear JF, Guanieri T, et al. Cesium chloride-induced long QT syndrome: demonstration of afterdepolarisations and triggered activity in syndrome: demonstration of afterde
vivo. Circulation 1985;72:1092-103.

21 Tzivoni D, Keren A, Cohen AM, et al. Magnesium therapy for torsade de pointes. Am J Cardiol 1984;53:528-30.

22 Zwillinger L. Ueber die Magnesiumwirkung auf das Herz. Klin Wochenschr 1935;14:1429-33

23 Khan MM, Logan KR, McComb JM, Adgey AAJ. Management of recurrent ventricular tachyarrhythmias associated with QT prolongation. Am J Cardiol 1981;47:1301-8.

24 Bronsky D, Dubin A, Kushner DS, et al. Calcium and the electrocardiogram III. The relationship of the intervals of the electrocardiogram to the levels of serum calcium. Am J Cardiol 1961;7:840-3.

25 Brachmann J, Scherlag BJ, Rosenshtraukh LV, Lazzara R. Bradycardiadependent triggered activity: relevance to drug-induced multiform ventricular tachycardia. Circulation 1983;68:846-56.

26 Hanich RF, Levine JH, Spera JF, Moore EN. Autonomic modulation of ventricular arrhythmia in cesium-chloride-induced long QT syndrome. Circulation 1988;77:1149-61.

27 Cranefield PF. Action potentials, after potentials and arrhythmias. Circ Res 1977;41:415-23.

28 Weintraub RG, Gow RM, Wilkinson JL. The congenital long QT syndromes in childhood. J Am Coll Cardiol 1990;16:674-80.

29 Schoenenberger RA, Haefeli WE, Weiss P, Ritz RF. Association of intravenous erythromycin and potentially fatal ventricular tachycardia with Q-T prolongation (torsade de pointes). BMJ 1990;300:1375-6.

30 Stein KM, Haronian H, Mensah GA, Acosta A, Jacobs J, Kligfield P. Ventricular tachycardia and torsade de pointes complicating pentamidine therapy of pneumocystis carinii pneumonia in the acquired immunotherapy of pneumocystis carinii pneumonia in the
deficiency syndrome. Am J Cardiol 1990;66:888-9.

31 Dessertenne F. Ordre ou chaos en électrocardiographie. Nouv Presse Méd $1973 ; 2: 1511-2$.

\section{Is there chaos in cardiology?}

A recent paper stated that "the health care system in the United States is in chaos." Though I am sure readers know what was meant, we have to be careful these days about the definition of chaos. Consulting a medical dictionary is unhelpful because there, chaos (Pelomyxa carolinensis) is an amoeba. ${ }^{2}$ Descriptions in Webster " ("a state of things in which chance is supreme . . . nature that is subject to no law . . . a state of utter confusion completely wanting in order, sequence, organisation or predictable operation") do not quite cover the modern, mathematical redefinition of chaos.

The new chaos is characterised by deterministic behaviour, where irregular patterns obey mathematical equations and are critically dependent on initial conditions. ${ }^{4}$ Modern chaos theory has been applied to a wide variety of biological phenomena ${ }^{5}$ and has become quite fashionable. In certain circles you are regarded as a barbarian if your conversation is not garnished with terms such as Hopf bifurcation points, Liapunov numbers, phase locking, or fractals.

Chaos theory has been used in two areas of cardiac electrophysiology: (a) the behaviour of cardiac tissue, or isolated myocytes, during repetitive stimulation and $(b)$ fibrillation and prefibrillatory states.

Chaos is irregular behaviour occurring in a nonlinear dynamic system. Cardiac cells may have several nonlinear, time-dependent variables (for instance supernormal excitability) and may exhibit irregular response patterns at a certain stimulation frequency. ${ }^{67}$ When isolated sheep Purkinje fibres exposed to a solution containing $7 \mathrm{mmol} / 1$ $\mathrm{K}^{+}$are repetitively stimulated at progressively faster rates, the so-called devil's staircase is seen, where $1: 1$ responses change into $2: 1$ and $3: 1$, with intermediate Wenckebach periodicity between the $1: 1$ and $2: 1$, and $2: 1$ and $3: 1$ responses. No irregular activity occurs. When, however, the extracellular $\mathrm{K}^{+}$concentration is lowered to $4 \mathrm{mmol} / \mathrm{l}$, and the fibres exhibit a brief period of supernormal excitability during the repolarisation phase, deterministic chaotic behaviour occurs at certain cycle lengths: either the stimulus-response pattern shows complex irregularities where no sequence is ever repeated in exactly the same way (this occurs at cycle lengths around $200 \mathrm{~ms}$ ), or at cycle lengths of $50 \mathrm{~ms}$ the relation between cycle length and action potential amplitude is chaotic. 'This example is chosen to show, on the one hand, that chaotic behaviour can indeed be induced in cardiac tissue, and that some satisfaction can be derived from the consideration that a unifying concept, chaos theory, links the stimulusresponse patterns in cardiac cells with other phenomena such as growth during embryonic development ${ }^{8}$ or the activity of phrenic nerves during mechanical ventilation of anaesthetised, paralysed cats. ${ }^{5}$ On the other hand, the conditions in which chaotic activity arises are extreme: artificial stimulation of isolated tissue at a certain $\mathrm{K}^{+}$ concentration at unphysiological frequencies.

Can chaotic behaviour be detected in "naturally" occurring rhythms? The obvious candidate for study is ventricular fibrillation, which traditionally has been called chaotic in the Webster sense. The identification of chaos in an existing rhythm is difficult. One technique is power spectrum analysis. A broad-band power spectrum is associated with chaos; however, ordinary random noise has a broad power spectrum as well. The power spectrum of electrocardiographic recordings during ventricular fibrillation is narrow ${ }^{910}$ and Kaplan and Cohen concluded from their analysis that "there is little utility in classifying fibrillation as chaotic." ${ }^{10}$ Would it be useful if fibrillation, or any other rhythm or conduction disorder, could be classified as chaotic? In this respect, the investigators in the chaotic domain are very enthusiastic: "A finding that ventricular fibrillation is chaos would suggest that there is a single mechanism at work in VF, and would provide guidance in the search for clinical precursors of VF"; 10 "... such new theories may help to strengthen the ties between the basic scientist and the cardiologist by opening new research avenues which may lead to the disclosure of the fundamental mechanisms of severe cardiac arrhythmias 